\title{
HSP90 inhibitor, AUY922, debilitates intrinsic and acquired lapatinib-resistant HER2-positive gastric cancer cells
}

\author{
Kang-Seo Park ${ }^{1,2}$, Yong Sang Hong ${ }^{1}$, Junyoung Choi ${ }^{1}$, Shinkyo Yoon ${ }^{1}$, Jihoon Kang ${ }^{1,3}$, Deokhoon Kim ${ }^{4}$, Kang-Pa Lee ${ }^{2}$, \\ Hyeon-Su Im ${ }^{5}$, Chang Hoon Lee ${ }^{6}$, Seyoung Seo ${ }^{1}$, Sang-We Kim ${ }^{1}$, Dae Ho Lee ${ }^{1, *} \mathcal{E}^{\circ}$ Sook Ryun Park ${ }^{1, *}$ \\ ${ }^{1}$ Department of Oncology, Asan Medical Center, University of Ulsan College of Medicine, Seoul 05505, ${ }^{2}$ Department of Biomedical \\ Sciences, University of Ulsan College of Medicine, Seoul 05505, ${ }^{3}$ Division of Hematology/Oncology, Department of Internal Medicine, \\ Kangbuk Samsung Hospital, Sungkyunkwan University School of Medicine, Seoul 03181, ${ }^{4}$ Asan Institute for Life Science, Department of \\ Pathology, Asan Medical Center, Seoul 05505, ${ }^{5}$ Department of Internal Medicine, Asan Medical Center, University of Ulsan College of \\ Medicine, Seoul 05505, ${ }^{6}$ Bio \& Drug Discovery Division, Center for Drug Discovery Technology, Korea Research Institute of Chemical \\ Technology (KRICT), Daejeon 34114, Republic of Korea
}

\begin{abstract}
Human epidermal growth factor receptor 2 (HER2) inhibitors, such as trastuzumab and lapatinib are used to treat HER2-positive breast and gastric cancers. However, as with other targeted therapies, intrinsic or acquired resistance to HER2 inhibitors presents unresolved therapeutic problems for HER2-positive gastric cancer. The present study describes investigations with AUY922, a heat shock protein 90 (HSP90) inhibitor, in primary lapatinib-resistant (ESO26 and OE33) and lapatinib-sensitive gastric cancer cells (OE19, N87, and SNU-216) harboring HER2 amplification/over-expression. In order to investigate whether AUY922 could overcome intrinsic and acquired resistance to HER2 inhibitors in HER2-positive gastric cancer, we generated lapatinib-resistant gastric cancer cell lines (OE19/LR and N87/LR) by continuous exposure to lapatinib in vitro. We found that activation of HER2 and protein kinase B (AKT) were key factors in inducing intrinsic and acquired lapatinib-resistant gastric cancer cell lines, and that AUY922 effectively suppressed activation of both HER2 and AKT in acquired lapatinib-resistant gastric cancer cell lines. In conclusion, AUY922 showed a synergistic anti-cancer effect with lapatinib and sensitized gastric cancer cells with intrinsic resistance to lapatinib. Dual inhibition of the HSP90 and HER2 signaling pathways could represent a potent therapeutic strategy to treat HER2-positive gastric cancer with intrinsic and acquired resistance to lapatinib. [BMB Reports 2018; 51(12): 660-665]
\end{abstract}

*Corresponding authors. Dae Ho Lee, Tel: +82-2-3010-3214; Fax: +82-2-3010-6961; E-mail: leedaeho@amc.seoul.kr; Sook Ryun Park, Tel: +82-2-3010-3206; Fax: +82-2-3010-6954; E-mail: srpark@ amc.seoul.kr

https://doi.org/10.5483/BMBRep.2018.51.12.259

Received 9 November 2018, Revised 15 November 2018, Accepted 28 November 2018

Keywords: AUY922, Drug resistance, Gastric cancer, HER2, Lapatinib

\section{INTRODUCTION}

Gastric cancer was the fifth most common cancer in 2018, and the third most common cause of cancer-related deaths in the world (1). South Korea had the highest rate of gastric cancer worldwide in 2018 (1). While surgery remains a major curative treatment modality for localized gastric cancer, palliative chemotherapy is recommended for patients with unresectable gastric cancer to prolong survival and improve quality of life (2). However, the median overall survival in metastatic gastric cancer patients is less than 12 months with the standard combination chemotherapy of fluoropyrimidine and platinum, indicating the need for more effective therapy $(3,4)$. Human epidermal growth factor receptor 2 (HER2) inhibitors, such as trastuzumab and lapatinib, have been developed for gastric and breast cancers harboring HER2 amplification/over-expression. Following success for breast cancer treatment, and a randomized phase III clinical trial investigating the survival benefit of combining trastuzumab with chemotherapy in HER2-positive gastric cancer patients (ToGA) (5), trastuzumab, a humanized monoclonal antibody targeting HER2 was also approved for unresectable or metastatic HER2-positive gastric cancer. In contrast, lapatinib, a dual HER2 and human epidermal growth factor receptor (EGFR) tyrosine kinase inhibitor did not demonstrate overall survival benefit, despite improvement in the objective response rate or progression-free survival in two phase III studies for HER2-positive gastric cancer, in first-line (LOGiC) and second-line (TyTAN) settings $(6,7)$. These unsatisfactory efficacy outcomes suggest the presence of drug resistance mechanisms or alternative pathways of escape from lapatinib in gastric cancer. Although the molecules that render intrinsic or acquired resistance to lapatinib are not well known, reports have suggested that lapatinib-resistance mechanisms involve FOXO1 suppression (8) and activation of alternative signaling pathways, such as MET (9), Testican-1 (10), AXL (11), HER2 (12-14) and AKT $(15,16)$, which induce bypass of HER2

ISSN: 1976-670X (electronic edition)

Copyright (c) 2018 by the The Korean Society for Biochemistry and Molecular Biology

() This is an open-access article distributed under the terms of the Creative Commons Attribution Non-Commercial License (http://creativecommons.org/licenses/by-nc/4.0) which permits unrestricted non-commercial use, distribution, and reproduction in any medium, provided the original work is properly cited. 
inhibition.

AUY922, luminespib, is a representative heat shock protein 90 (HSP90) inhibitor and shows anti-cancer effects via binding to the ATPase domain of HSP90, causing loss of chaperone functions. HSP90 inhibitor-misfolded client proteins, including HER2, EGFR, IGF1R, AKT, RAF-1, IKK, c-Kit, v-SRC, NPM-ALK, BCR-ABL, p53, STAT3, HIF1, and CDK4/6, are degraded by proteasomes $(17,18)$. This process has been shown to induce pro-apoptosis and anti-proliferation effects in various tumors (19-23). AUY922 monotherapy showed promising activity in non-small cell lung cancers harboring EGFR exon 20 insertions (24), and various stages of preclinical and clinical development as a component of combination therapies have been performed $(19,25)$.

AUY922 has been shown to inhibit growth and proliferation by significantly decreasing AKT and ERK, as well as HER2 levels in HER2-positive breast cancer and gastric cancer cell lines $(16,21,23)$. Furthermore, combination of AUY922 (16) or 17-AAG, another HSP90 inhibitor (26) with trastuzumab, showed promising antitumor activity in HER2-positive trastuzumab-resistant breast and gastric cancer preclinical models or metastatic breast cancer patients. These results suggest HSP90 inhibition could be a treatment strategy to overcome resistance to HER2-targeted therapies. However, the effect of AUY922 on HER2-positive and lapatinib-resistant gastric cancer remains to be investigated.

In this study, we describe the dramatically synergistic
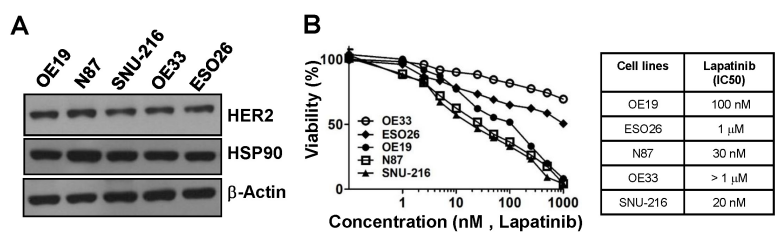

C

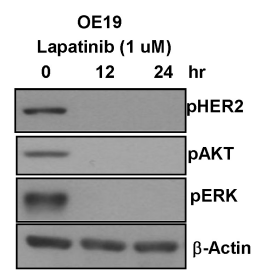

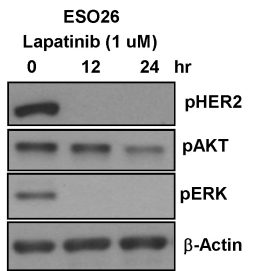

Fig. 1. In vitro response to lapatinib in HER2-positive gastric cancer cells. (A) Baseline expression of HER2, HSP90, and $\beta$-Actin measured by Western blotting in five parental HER2-positive gastric cancer cells. (B) The anti-cancer effects of lapatinib alone in five HER2-positive gastric cancer cell lines. Cell proliferation was measured by the CellTiter-Glo luminescent cell viability assay. The average results $( \pm S D)$ of three independent experiments are shown. (C and D) Effects on the downstream pathway by lapatinib alone. Western blotting of pHER2, pAKT, and PERK levels following treatment with lapatinib in lapatinib-sensitive HER2-positive gastric cancer cells (OE19) and intrinsic lapatinib-resistant HER2-positive gastric cancer cells (ESO26). $\beta$-Actin was included as a loading control. anti-cancer effect of AUY922, an HSP90 inhibitor in combination with lapatinib, in intrinsic and acquired lapatinibresistant gastric cancer cell lines harboring HER2 amplification/ over-expression.

\section{RESULTS}

AKT activation bypasses HER2 inhibition in HER2-positive gastric cancer cells with intrinsic resistance to lapatinib

Western blotting was performed to determine the expression of HER2 and assess signaling pathways in five gastric cancer cell lines. Interestingly, although HER2 was highly expressed and lapatinib inhibited the cell growth in a concentrationdependent manner in all cell lines (Fig. 1A), sensitivity to lapatinib was greater in OE19, N87 and SNU-216 cells, than in ESO26 and OE33 cells. Lapatinib significantly inhibited the growth of OE19, N87 and SNU-216 cells, but not ESO26 and OE33 cell lines in a concentration-dependent manner (Fig. 1B). Whereas lapatinib remarkably inhibited pAKT in a lapatinib-sensitive OE19 cell line, AKT activation persisted even after lapatinib treatment in an intrinsic lapatinib-resistant ESO26 cell line (Fig. 1C and 1D).

\section{Both intrinsic lapatinib-resistant and lapatinib-sensitive gastric cancer cells are all sensitive to AUY922 via suppression of HER2 and AKT activation} To determine the IC50 of AUY922 in HER2-positive gastric
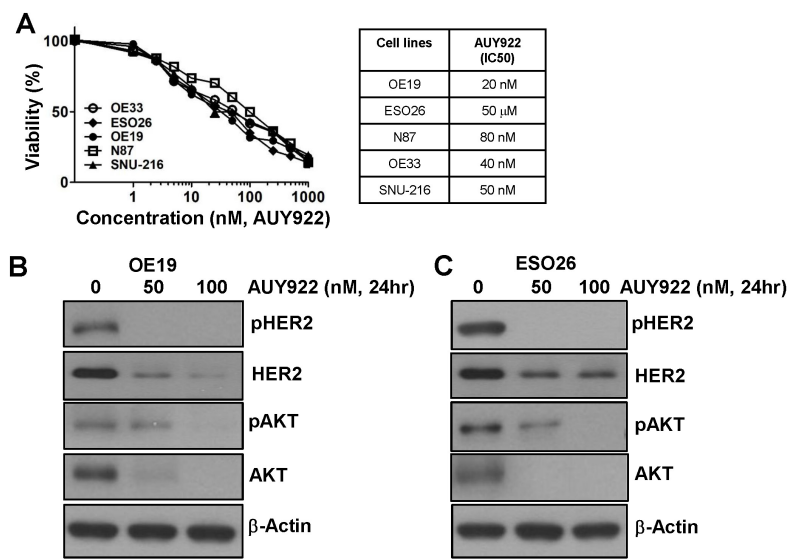

Fig. 2. Both intrinsic lapatinib-resistant and lapatinib-sensitive gastric cancer cells are sensitive to AUY922 via suppression of HER2 and AKT activation. (A) The anti-cancer effects of AUY922 alone in five HER2-positive gastric cancer cell lines. Cell proliferation was measured by the CellTiter-Glo luminescent cell viability assay. The average results $( \pm S D)$ of three independent experiments are shown. (B and C) The effects on the downstream pathway by lapatinib alone. Western blotting of pHER2, HER2, pAKT, and AKT levels following treatment with AUY922 in lapatinib-sensitive HER2-positive gastric cancer cells (OE19) and intrinsic lapatinib-resistant HER2-positive gastric cancer cells (ESO26). $\beta$-Actin was included as a loading control. 
cancer cells, five gastric cancer cells, OE19, ESO26, N87, OE33 and SNU-216, were exposed to AUY922 at concentrations ranging from $1 \mathrm{nM}$ to $1 \mu \mathrm{M}$. AUY922 showed the potent anti-cancer effect, with low IC50 values in both intrinsic lapatinib-resistant and lapatinib-sensitive gastric cancer cells, though AUY922 did not kill these cancer cells completely, even at $1 \mu \mathrm{M}$ (Fig. 2A).

To assess if AUY922 could block HER2 and AKT activation, Western blot analyses were performed to measure pHER, HER2, pAKT, and AKT expression in OE19, and ESO26 cells after exposure to $50 \mathrm{nM}$ and $100 \mathrm{nM}$ AUY922 for $24 \mathrm{hrs}$. AUY922 effectively and equally suppressed pHER2, as well as pAKT expressions in both lapatinib-sensitive OE19 (Fig. 2B) and intrinsic lapatinib-resistant ESO26 cells (Fig. 2C).

\section{Activation of HER2 and AKT bypass HER2 inhibition in HER2-positive gastric cancer cells with acquired resistance to lapatinib}

To establish lapatinib-resistant gastric cancer cells, we exposed OE19 and N87 cells to increasing concentrations of lapatinib in culture over a 3-month period. We then treated these resistant cells and parental cells with concentrations of lapatinib ranging from $1 \mathrm{nM}$ to $1 \mu \mathrm{M}$. Lapatinib-resistant
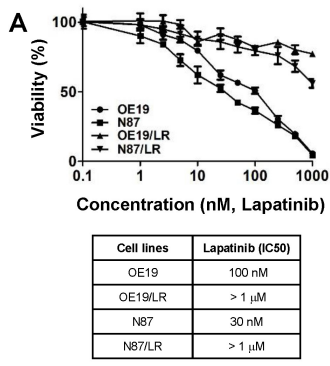

B

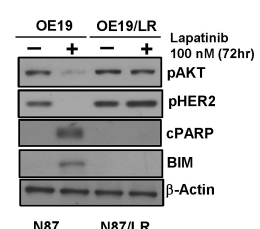

C
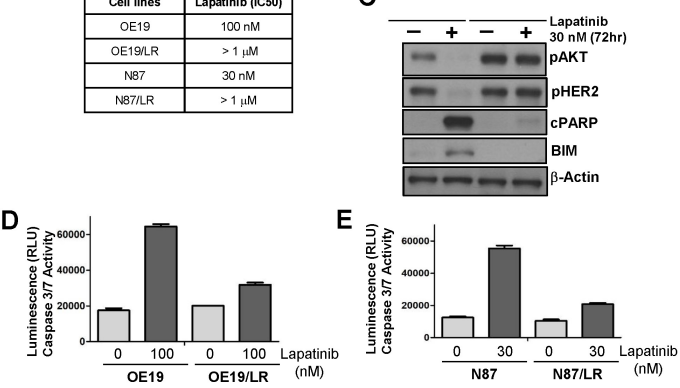

Fig. 3. Activation of HER2 and AKT bypass HER2 inhibition in HER2-positive gastric cancer cells with acquired resistance to lapatinib. (A) The anti-cancer effect of lapatinib in two parental HER2-positive gastric cancer cells, OE19 and N87, and acquired lapatinib-resistant HER2-positive gastric cancer cells, OE19/LR and N87/LR. Cell proliferation was measured by the CellTiter-Glo luminescent cell viability assay. The average results $( \pm$ SD) of three independent experiments are shown. ( $B$ and $C$ ) Expression of pHER2, pAKT, cPARP, and BIM was determined following 72 $h$ incubation with increasing doses of lapatinib in OE19, OE19/LR, N87, and N87/LR cell lines. $\beta$-Actin was included as a loading control. (D and E) Caspase 3/7 activity of parental cells (OE19 and N87) and acquired lapatinib-resistant cells (OE19/LR and N87/LR) after treatment with $100 \mathrm{nM}$ lapatinib.
OE19/LR and N87/LR cells exhibited higher IC50 than their OE19 and N87parental cells, in response to lapatinib (Fig. 3A). AKT and HER2 phosphorylation in lapatinib-resistant OE19/LR and N87/LR cells was not inhibited by lapatinib treatment, in contrast to lapatinib-sensitive OE19 and N87 cells. This implies that activation of AKT and HER2 signaling may play a role in lapatinib resistance (Fig. $3 \mathrm{~B}$ and $3 \mathrm{C}$ ). Caspase 3/7 assays revealed that these two resistant cells attenuated lapatinib-induced apoptosis (Fig. 3D and 3E).

\section{AUY922 sensitizes HER2-positive gastric cancer cells with acquired resistance to lapatinib}

We assessed the effect of the combination of AUY922 and lapatinib for 72 hrs in OE19/LR and N87/LR cells. Western blot analysis revealed that both pHER2 and pAKT expressions were downregulated more in cells exposed to the drug combination, than in cells exposed to either AUY922 or lapatinib alone (Fig. 4A). Levels of cleaved PARP, an apoptosis marker, were also elevated to a higher extent by the combination treatment, than by either drug alone (Fig. 1A). To determine if AUY922 could overcome acquired resistance to lapatinib and synergize with lapatinib in OE19/LR and N87/LR cells, we exposed two cell lines to different concentrations of AUY922 alone, lapatinib alone, and their combinations. In cell

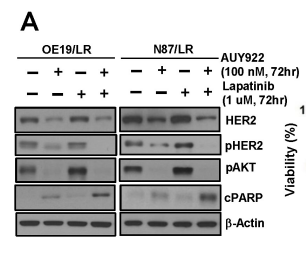

D
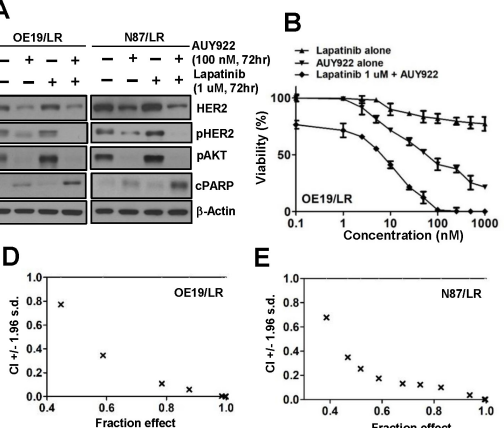

C
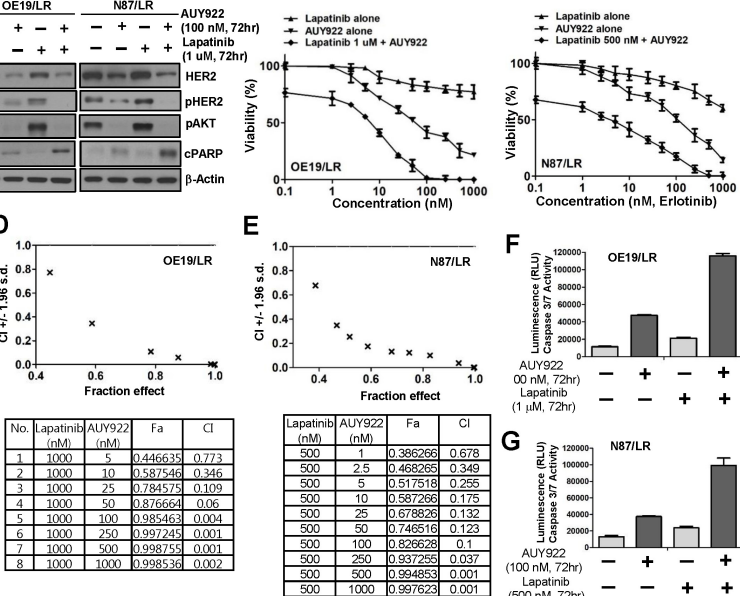

Fig. 4. AUY922 sensitizes HER2-positive gastric cancer cells with acquired resistance to lapatinib. (A) Effect of the combination of lapatinib and AUY922 on downstream signaling. Western blotting of HER2, pHER2, pAKT, and CPARP following treatment with lapatinib and AUY922 for $72 \mathrm{~h}$ in OE19/LR and N87/LR cell lines. (B and C) Synergistic effect of the combination of lapatinib and AUY922. Proliferation assays were performed in OE19/LR and N87/LR cells treated with $1 \mu \mathrm{M}$ or $500 \mathrm{nM}$ of lapatinib, plus increasing concentrations of AUY922 from $1 \mathrm{nM}$ to $1 \mu \mathrm{M}$ for 3 days. ( $\mathrm{D}$ and $\mathrm{E}$ ) $\mathrm{Cl}$, combination index, and $\mathrm{Cl}$ table of $\mathrm{B}$ and $\mathrm{C}$. ( $F$ and $G$ ) Caspase 3/7 activity of acquired lapatinib-resistant cells (OE19/LR and N87/LR) after single-agent (AUY922 or lapatinib) or combination (AUY922 plus lapatinib) treatment. 
viability studies, the combination of AUY922 with lapatinib at $1 \mu \mathrm{M}$ or $500 \mathrm{nM}$ showed significant synergy in OE19/LR (Fig. 4B) and N87/LR cells (Fig. 4C), respectively, compared with either agent alone. In addition, we confirmed these synergistic effects using the CalcuSyn program (Biosoft, UK) (Fig. 4D and $4 \mathrm{E})$. These observed synergistic effects occurred via increased caspase-3/7 activity (Fig. 4F and $4 \mathrm{G}$ ). Taken together, these results indicate that dual targeting of HER2 and HSP90 overcomes acquired resistance to HER2 inhibition by lapatinib, in vitro.

\section{DISCUSSION}

Although the prognosis for HER2-positive advanced gastric cancer patients has been significantly improved since the development of anti-HER2 targeted therapy, such as trastuzumab (5), appearance of primary or acquired resistance to anti-HER2 therapy remains a major therapeutic challenge. While trastuzumab was established as standard therapy for both HER2-positive breast cancer and gastric cancer $(5,27)$, other anti-HER2 targeting agents, such as lapatinib, trastuzumab emtansine, and pertuzumab have shown different efficacy outcomes in clinical trials of both cancers, resulting in approval for their use only in breast cancer. Lapatinib enhanced antitumor efficacy in terms of overall response rate or progression-free survival, when combined with cytotoxic chemotherapy in HER2-positive metastatic gastric cancer, but this did not translate to an overall survival benefit $(6,7)$. The failure of gastric cancer clinical trials for lapatinib might have been caused by inadequate patient selection, HER2 heterogeneity, or statistically under-powered study design. However, one of the most important reasons may be intrinsic or acquired resistance to lapatinib in gastric cancer. Considering multiple escape mechanisms that circumvent inhibition of the HER2 signaling pathways, including compensatory activation of the HER network or activation of other redundant survival pathways (8-16), the combination strategy to block these escape mechanisms could be pursued to overcome resistance to lapatinib in HER2-positive gastric cancer.

In this study, we focused on the combination of an HSP90 inhibitor with lapatinib for treatment of acquired lapatinibresistant HER2-positive gastric cancer cells. HSP90 is highly expressed in various tumors, including HER2-positive gastric cancers, and has been significantly associated with poor prognosis $(28,29)$. HSP90 is a molecular chaperone that acts to stabilize client proteins, many of which are oncoproteins, including HER2 $(19,20,30)$. HSP90 is a good therapeutic target in HER2-positive cancer because it is in charge of protein stabilization of multiple proteins involved in tumor progression, as well as HER2. The HSP90 inhibitor AUY922 induces degradation of HER2 via ubiquitinylation and lysosomal pathways in proteasomes (31). Preclinical data have suggested that AUY922 could play a role in overcoming resistance to trastuzumab in HER2-positive breast and gastric cancer cells $(16,32)$. Besides clinical antitumor activity in non-small cell lung cancer, where AUY922 showed promising activity in cases harboring EGFR exon 20 insertions (24) or ALK rearrangements (33), it also showed encouraging antitumor efficacy when combined with trastuzumab to treat metastatic HER2-positive breast cancer patients who had progressed on trastuzumab-based therapy (34). However, the potential of AUY922 in combination with lapatinib to overcome lapatinib-resistant HER2-positive gastric cancer cells has not yet been investigated.

In this research, we are the first to describe the potent anti-proliferative effects of AUY922 in two intrinsic lapatinib-resistant cells (OE33 and ESO26), as well as in lapatinib-sensitive HER2-positive gastric cancer cell lines (OE19, N87 and SNU-216). In addition, we report that AUY922 markedly decreased the levels of HER2 and AKT in both lapatinib-sensitive and lapatinib-resistant cell lines. These results suggest that AUY922 could be useful in treatment of intrinsic lapatinib-resistant HER2-positive gastric cancer.

We also investigated if AUY922 was effective in two acquired lapatinib-resistant HER2-positive gastric cancer cell lines OE19/LR and N87/LR. The acquired lapatinib-resistant cell lines were highly sensitive to AUY922, though cells were not completely killed, even at the highest concentration used $(1 \mu \mathrm{M})$. In our study, lapatinib-resistant cells were still dependent on the HER2 signaling pathways, including the AKT pathway. Therefore, the combination of AUY922 with lapatinib showed a dramatic synergistic effect in OE19/LR and N87/LR cell lines.

In summary, our results support clinical development of AUY922 as a treatment strategy for overcoming intrinsic or acquired resistance to lapatinib in HER2-positive gastric cancer.

\section{MATERIALS AND METHODS}

\section{Cell culture and reagents}

The human gastric cell lines OE19, OE33, N87, ESO26, and SNU-216 were purchased from the American Type Culture Collection (Manassas, VA, USA) and Korea cell line bank (Seoul, Korea). These cell lines were grown at $37^{\circ} \mathrm{C}$ in $5 \% \mathrm{CO}_{2}$ in RPMI-1640 and DEME, containing 10\% fetal bovine serum from GIBCO (Waltham, MA, USA). NVP-AUY922 and lapatinib were purchased from Selleck Chemicals (Houston, TX, USA), dissolved in DMSO to a final concentration of 10 $\mathrm{mmol} / \mathrm{L}$, and stored at $-20^{\circ} \mathrm{C}$.

\section{Cell viability assay}

Cell viability was measured using the CellTiter-Glo luminescent cell viability assay (Promega, Madison, WI, USA), following the manufacturer's instructions. Approximately $3 \times$ $10^{3}$ cells were transferred to white 96 well plates. The next day, the culture medium was removed, and the desired 
concentrations of AUY922 or/and lapatinib (CP358774) were added to a volume of $100 \mu \mathrm{l}$. After $72 \mathrm{~h}, 100 \mu \mathrm{l}$ of CellTiter-Glo reagent was added, and the plates were incubated for $10 \mathrm{~min}$ at room temperature. The luminescence was measured using a Wallac 1420 apparatus (PerkinElmer, Boston, MA, USA)

\section{Western blot analysis}

Cells were suspended in modified RIPA lysis buffer $(150 \mathrm{mM}$ $\mathrm{NaCl}, 1 \mathrm{mM}$ EDTA, 1\% Triton X-100, 1\% NP-40, 0.5\% sodium deoxycholate, $0.1 \% \mathrm{SDS}$, and $50 \mathrm{mM}$ Tris- $\mathrm{HCl}[\mathrm{pH}$ 7.4]), containing a protease inhibitor cocktail (Roche, Mannheim, Germany) and phosphatase inhibitors (1 $\mathrm{mM}$ sodium fluoride and $2 \mathrm{mM}$ sodium orthovanadate) on ice for $30 \mathrm{~min}$, and centrifuged at $15,000 \times \mathrm{g}$ for $30 \mathrm{~min}$ to collect whole cell lysates. The proteins (10-20 $\mu \mathrm{g})$ were separated on an $8 \%-12 \%$ SDS-PAGE and transferred onto a PVDF membrane (Millipore, Bedford, MA, USA). Western blotting was performed with specific primary antibodies and peroxidase-conjugated anti-mouse or anti-rabbit secondary antibodies. Proteins were visualized with ECL Plus enhanced chemiluminescence reagents (Amersham Biosciences, Piscataway, NJ, USA). The commercial antibodies used in this study included HER2, pHER2, HSP90, AKT, pAKT, pERK, CPARP, BIM, and $\beta$-actin (Cell Signaling Technology, Danvers, MA, USA).

\section{Caspase $3 / 7$ assay}

Caspase 3 and 7 activations were measured using the Caspase-Glo 3/7 Luminescence Assay (Promega Corp., Madison, WI, USA), following the manufacturer's protocol. Protein samples from cells were prepared using RIPA buffer in the same manner as Western blot sample preparation. Ten micrograms of protein samples in $100 \mu \mathrm{l}$ total volume were transferred to white 96 wells, and $100 \mu \mathrm{l}$ of equilibrated Caspase-Glo 3/7 reagent was added to protein samples and incubated for $1 \mathrm{~h}$ at room temperature. Luminescence was measured using the Wallac 1420 apparatus (PerkinElmer, Waltham, MA, USA).

\section{Calculation of the combination index}

Activity of the drugs, used singly or in combination treatment, was estimated using CalcuSyn software (Biosoft, Ferguson, MO, USA). Briefly, this program calculated and determined the combination index, a quantitative measure of the degree of drug interaction. A combination index $(\mathrm{Cl})<1, \mathrm{Cl}=1$, and $\mathrm{Cl}>1$ indicated synergistic, additive, and antagonistic effects, respectively.

\section{ACKNOWLEDGEMENTS}

This manuscript was supported by a grant (2014-599) from the Asan Institute for Life Sciences, Asan Medical Center, Seoul. Korea and a grant of the Basic Science Research Program of the National Research Foundation of Korea (NRF) funded by the Ministry of Education (No. 2017R1D1A1B03033550) and a grant of the Korea Health Technology R\&D Project through the Korea Health Industry Development Institute (KHIDI), funded by the Ministry of Health \& Welfare, Republic of Korea (grant number : HI15C0972).

\section{CONFLICTS OF INTEREST}

Dea Ho Lee declares honoraria from AstraZeneca, Boehringer Ingelheim, Bristol-Myers Squibb, CJ HealthCare, Eli Lilly and Company, Janssen Pharmaceutica, Merck \& Co., MSD, Mundipharma, Novartis, Ono Pharmaceutical Co., Ltd., Pfizer, Roche, Samyang Biopharmaceuticals, and ST Cube. The other authors declare no conflict of interest.

\section{REFERENCES}

1. Bray F, Ferlay J, Soerjomataram I, Siegel RL, Torre LA and Jemal A (2018) Global cancer statistics 2018: GLOBOCAN estimates of incidence and mortality worldwide for 36 cancers in 185 countries. CA Cancer J Clin 68, 394-424

2. Glimelius B, Ekstrom K, Hoffman K et al (1997) Randomized comparison between chemotherapy plus best supportive care with best supportive care in advanced gastric cancer. Ann Oncol 8, 163-168

3. Cunningham D, Starling N, Rao S et al (2008) Capecitabine and oxaliplatin for advanced esophagogastric cancer. N Engl J Med 358, 36-46

4. Kang YK, Kang WK, Shin DB et al (2009) Capecitabine/cisplatin versus 5-fluorouracil/cisplatin as first-line therapy in patients with advanced gastric cancer: a randomised phase III noninferiority trial. Ann Oncol 20, 666-673

5. Bang YJ, Van Cutsem E, Feyereislova A et al (2010) Trastuzumab in combination with chemotherapy versus chemotherapy alone for treatment of HER2-positive advanced gastric or gastro-oesophageal junction cancer (ToGA): a phase 3, open-label, randomised controlled trial. Lancet 376, 687-697

6. Hecht JR, Bang YJ, Qin SK et al (2016) Lapatinib in Combination With Capecitabine Plus Oxaliplatin in Human Epidermal Growth Factor Receptor 2-Positive Advanced or Metastatic Gastric, Esophageal, or Gastroesophageal Adenocarcinoma: TRIO-013/LOGiC-A Randomized Phase III Trial. J Clin Oncol 34, 443-451

7. Satoh T, Xu RH, Chung HC et al (2014) Lapatinib plus paclitaxel versus paclitaxel alone in the second-line treatment of HER2-amplified advanced gastric cancer in Asian populations: TyTAN--a randomized, phase III study. J Clin Oncol 32, 2039-2049

8. Park J, Choi Y, Ko YS et al (2018) FOXO1 Suppression is a Determinant of Acquired Lapatinib-Resistance in HER2Positive Gastric Cancer Cells Through MET Upregulation. Cancer Res Treat 50, 239-254

9. Chen CT, Kim H, Liska D, Gao S, Christensen JG and Weiser MR (2012) MET activation mediates resistance to lapatinib inhibition of HER2-amplified gastric cancer cells. Mol Cancer Ther 11, 660-669 
10. Kim HP, Han SW, Song SH et al (2014) Testican-1mediated epithelial-mesenchymal transition signaling confers acquired resistance to lapatinib in HER2-positive gastric cancer. Oncogene 33, 3334-3341

11. Liu L, Greger J, Shi H et al (2009) Novel mechanism of lapatinib resistance in HER2-positive breast tumor cells: activation of AXL. Cancer Res 69, 6871-6878

12. Trowe T, Boukouvala S, Calkins K et al (2008) EXEL-7647 inhibits mutant forms of ErbB2 associated with lapatinib resistance and neoplastic transformation. Clin Cancer Res $14,2465-2475$

13. Xu X, De Angelis C, Burke KA et al (2017) HER2 Reactivation through Acquisition of the HER2 L755S Mutation as a Mechanism of Acquired Resistance to HER2-targeted Therapy in HER2(+) Breast Cancer. Clin Cancer Res 23, 5123-5134

14. D'Amato V, Raimondo L, Formisano L et al (2015) Mechanisms of lapatinib resistance in HER2-driven breast cancer. Cancer Treat Rev 41, 877-883

15. Hegde PS, Rusnak D, Bertiaux M et al (2007) Delineation of molecular mechanisms of sensitivity to lapatinib in breast cancer cell lines using global gene expression profiles. Mol Cancer Ther 6, 1629-1640

16. Wainberg ZA, Anghel A, Rogers AM et al (2013) Inhibition of HSP90 with AUY922 induces synergy in HER2-amplified trastuzumab-resistant breast and gastric cancer. Mol Cancer Ther 12, 509-519

17. Lee KH, Lee JH, Han SW et al (2011) Antitumor activity of NVP-AUY922, a novel heat shock protein 90 inhibitor, in human gastric cancer cells is mediated through proteasomal degradation of client proteins. Cancer Sci $102,1388-1395$

18. Mayor-Lopez L, Tristante E, Carballo-Santana M et al (2014) Comparative Study of 17-AAG and NVP-AUY922 in Pancreatic and Colorectal Cancer Cells: Are There Common Determinants of Sensitivity? Transl Oncol 7, 590-604

19. Garcia-Carbonero R, Carnero A and Paz-Ares L (2013) Inhibition of HSP90 molecular chaperones: moving into the clinic. Lancet Oncol 14, e358-369

20. Tillotson B, Slocum K, Coco J et al (2010) Hsp90 (heat shock protein 90) inhibitor occupancy is a direct determinant of client protein degradation and tumor growth arrest in vivo. J Biol Chem 285, 39835-39843

21. Jensen MR, Schoepfer J, Radimerski T et al (2008) NVP-AUY922: a small molecule HSP90 inhibitor with potent antitumor activity in preclinical breast cancer models. Breast Cancer Res 10, R33

22. Gaspar N, Sharp SY, Eccles SA et al (2010) Mechanistic evaluation of the novel HSP90 inhibitor NVP-AUY922 in adult and pediatric glioblastoma. Mol Cancer Ther 9,
1219-1233

23. Eccles SA, Massey A, Raynaud FI et al (2008) NVP-AUY922: a novel heat shock protein 90 inhibitor active against xenograft tumor growth, angiogenesis, and metastasis. Cancer Res 68, 2850-2860

24. Piotrowska Z, Costa DB, Oxnard GR et al (2018) Activity of the Hsp90 inhibitor Luminespib Among Non-Small Cell Lung Cancers Harboring EGFR Exon 20 Insertions. Ann Oncol 29, 2092-2097

25. Seggewiss-Bernhardt R, Bargou RC, Goh YT et al (2015) Phase $1 / 1 \mathrm{~B}$ trial of the heat shock protein 90 inhibitor NVP-AUY922 as monotherapy or in combination with bortezomib in patients with relapsed or refractory multiple myeloma. Cancer 121, 2185-2192

26. Modi S, Stopeck A, Linden H et al (2011) HSP90 inhibition is effective in breast cancer: a phase II trial of tanespimycin (17-AAG) plus trastuzumab in patients with HER2-positive metastatic breast cancer progressing on trastuzumab. Clin Cancer Res 17, 5132-5139

27. Slamon DJ, Leyland-Jones B, Shak S et al (2001) Use of chemotherapy plus a monoclonal antibody against HER2 for metastatic breast cancer that overexpresses HER2. N Engl J Med 344, 783-792

28. Berezowska S, Novotny A, Bauer K et al (2013) Association between HSP90 and Her2 in gastric and gastroesophageal carcinomas. PLoS One 8, e69098

29. Wang J, Cui S, Zhang X, Wu Y and Tang H (2013) High expression of heat shock protein 90 is associated with tumor aggressiveness and poor prognosis in patients with advanced gastric cancer. PLoS One 8, e62876

30. Taipale M, Krykbaeva I, Koeva M et al (2012) Quantitative analysis of HSP90-client interactions reveals principles of substrate recognition. Cell 150, 987-1001

31. Raja SM, Clubb RJ, Bhattacharyya M et al (2008) A combination of Trastuzumab and 17-AAG induces enhanced ubiquitinylation and lysosomal pathway-dependent ErbB2 degradation and cytotoxicity in ErbB2-overexpressing breast cancer cells. Cancer Biol Ther 7, 1630-1640

32. Canonici A, Qadir Z, Conlon NT et al (2018) The HSP90 inhibitor NVP-AUY922 inhibits growth of HER2 positive and trastuzumab-resistant breast cancer cells. Invest New Drugs 36, 581-589

33. Felip E, Barlesi F, Besse B et al (2018) Phase 2 Study of the HSP-90 Inhibitor AUY922 in Previously Treated and Molecularly Defined Patients with Advanced Non-Small Cell Lung Cancer. J Thorac Oncol 13, 576-584

34. Kong A, Rea D, Ahmed S et al (2016) Phase 1B/2 study of the HSP90 inhibitor AUY922 plus trastuzumab in metastatic HER2-positive breast cancer patients who have progressed on trastuzumab-based regimen. Oncotarget 7, 37680-37692 